\title{
Erratum to: Methods of continuous translation of the origin of the current density revisited
}

\section{P. Lazzeretti}

Published online: 30 December 2012

(c) Springer-Verlag Berlin Heidelberg 2012

Erratum to: Theor Chem Acc (2012) 131:1222

DOI 10.1007/s00214-012-1222-y

In the original publication of the article, Eqs. (68), (84) and (85) are incorrect. The correct versions of these equations are given below

$$
\begin{aligned}
\mathbf{J}_{p}^{\mathbf{d} \times \mathbf{B}}(\mathbf{r})= & -\frac{n e}{m_{e}} \int \mathrm{d} \mathbf{x}_{2}, \ldots, \mathrm{d} \mathbf{x}_{n} \\
& \times\left[\mathbf{d} \times \mathbf{B} \cdot \Psi_{a}^{\mathbf{d} \times \mathbf{B} *}\left(\mathbf{r}, \mathbf{x}_{2}, \ldots, \mathbf{x}_{n}\right) \hat{\mathbf{p}} \Psi_{a}^{(0)}\left(\mathbf{r}, \mathbf{x}_{2}, \ldots, \mathbf{x}_{n}\right)\right. \\
& \left.+\Psi_{a}^{(0) *}\left(\mathbf{r}, \mathbf{x}_{2}, \ldots, \mathbf{x}_{n}\right) \hat{\mathbf{p}} \mathbf{d} \times \mathbf{B} \cdot \Psi_{a}^{\mathbf{d} \times \mathbf{B}}\left(\mathbf{r}, \mathbf{x}_{2}, \ldots, \mathbf{x}_{n}\right)\right] \\
\hat{\xi}_{\alpha \beta}^{\Delta}=- & \frac{i e^{2}}{8 m_{e} \hbar} \sum_{i=1}^{n}\left\{\epsilon_{\beta \gamma \delta}\left[r_{\gamma}, \hat{u}_{\delta \alpha}\right]_{i}+\epsilon_{\alpha \gamma \delta}\left[r_{\gamma}, \hat{u}_{\delta \beta}\right]_{i}\right\} \\
= & -\frac{i e^{2}}{8 m_{e} \hbar}\left\{\epsilon_{\beta \gamma \delta}\left[\hat{R}_{\gamma}, \hat{U}_{\delta \alpha}\right]+\epsilon_{\alpha \gamma \delta}\left[\hat{R}_{\gamma}, \hat{U}_{\delta \beta}\right]_{i}\right\} \\
\hat{\sigma}_{\alpha \beta}^{\Delta I}= & \frac{i e^{2}}{2 m_{e} \hbar} \epsilon_{\beta \gamma \delta} \sum_{i=1}^{n}\left[r_{\gamma}, \hat{t}_{I_{\delta \alpha}}\right]_{i} \\
= & \frac{i e^{2}}{2 m_{e} \hbar} \epsilon_{\beta \gamma \delta}\left[\hat{R}_{\gamma}, \hat{T}_{I_{\delta \alpha}}\right]
\end{aligned}
$$$$
\left.+\Psi_{a}^{(0) *}\left(\mathbf{r}, \mathbf{x}_{2}, \ldots, \mathbf{x}_{n}\right) \hat{\mathbf{p}} \mathbf{d} \times \mathbf{B} \cdot \Psi_{a}^{\mathbf{d} \times \mathbf{B}}\left(\mathbf{r}, \mathbf{x}_{2}, \ldots, \mathbf{x}_{n}\right)\right]
$$

The online version of the original article can be found under doi:10.1007/s00214-012-1222-y.

P. Lazzeretti $(\bowtie)$

Dipartimento di Chimica dell'Università degli Studi di Modena

e Reggio Emilia, Via Campi 183, 41124 Modena, Italy

e-mail: lazzeret@unimore.it 